\title{
Diversity and Convergence of Population Aging: Evidence from China and Canada
}

\author{
Long Mo \\ Statistics Canada \\ Ottawa, Ontario, Canada \\ Jacques Légaré \\ Département de démographie \\ Université de Montréal \\ Montréal, Québec, Canada
}

\begin{abstract}
Taking the diversity and the convergence of demographic transitions into consideration, it is hypothesized that population aging that occurs in developed countries and developing countries will reflect diversity, but will also show some convergence. In order to test this hypothesis, the present study compares the population aging experiences of China (1971-2050) and Canada (1911-2050) and places them within the context of the demographic transition. In this paper we learn how, as population ages, these two countries will, through two distinct pathways, arrive at similar age structures by the middle of the $21^{\text {st }}$ century. Both the diversity and the convergence of population aging are shown in this comparative study.
\end{abstract}

Key Words: Population aging, demographic transition, China, Canada 
Long Mo and Jacques Légaré

\section{Résumé}

En constatant la diversité et la convergence des transitions démographiques, nous faisons l'hypothèse que le processus du vieillissement démographique comporte également une certaine diversité et montrera une convergence, en particulier, entre les pays développés et les pays en développement. Afin de vérifier cette hypothèse, la présente étude compare les vieillissements démographiques en Chine (1971-2050) et au Canada (1911-2050), en les mettant dans le contexte de la transition démographique. Cet article montre qu'à mesure que vieillit la population, comment ces deux pays arriveront par deux voies distinctes à une structure par âge similaire au milieu du XXIème siècle. La diversité et la convergence du vieillissement sont toutes deux illustrées dans cette étude comparative.

Mots-clés: Vieillissement démographique, la transition démographique, China, Canada

\section{Introduction}

Without any doubt, we have entered into an era of globalization of population aging (Loriaux 2002). According to United Nations projections (1999b), during the first half of the $21^{\text {st }}$ century, in almost all countries, whether developed or developing, age structure changes will follow the same trend, that is, the aging of the population. It is a well-established fact that demographic aging is the natural outcome of demographic transition. Numerous quantitative analyses, including those about the Canadian and Chinese experiences, have shown that fertility transition was the major determinant of the age structure transition (United Nations 1988; George et al. 1991; Du 1994; Mo 2002). Taking into account the diversity in timing and process, but also the eventual convergence of demographic transitions (Chesnais 1992), it is hypothesized that population aging that occurs in developed countries and developing countries will reflect diversity but will also show convergence. To date, there have been few studies focusing on this issue. 
Diversity and Convergence of Population Aging:

Evidence from China and Canada

In order to test this hypothesis, the present study compares the population aging experiences of China (1971-2050) and Canada (1911-2050) and places them within the context of demographic transition. These two countries have been selected here because they represent the contrasting contexts of developed and developing countries, and Eastern and Western cultures, respectively. In addition, these two countries stand as two extreme examples of populations for which the demographic aging process is affected by population policy (i.e. by a family planning policy in China and by an immigration policy in Canada) and therefore show a tendency to converge (Mo and Légaré, 2003). Basic data are drawn from United Nations (1998; 1999a; 1999b) and Statistics Canada (1936; 1973) tabulations. While introducing a new approach, we will show how these two countries will achieve the same age transition (with the proportion of persons aged 60 and over in the total population passing from $7 \%$ to $30 \%$ during the periods studied) and will reach a similar age structure by the middle of the $21^{\text {st }}$ century, through two distinct pathways.

\section{Patterns of Population Aging}

Population aging can be characterized by its take off, its levels and its speed. Diversity and convergence of population aging can be observed by looking at the dissimilarities and the similarities of those components of population aging in China and Canada.

\section{The Take Off of Population Aging}

Usually, "population aging" in the modern sense of the term refers to the gradual increase, above a stated level, of the proportion of older people in a population. According to this concept, we infer that population aging started by 1911 in Canada and by 1971 in China, because since then and until 2050, the proportion of the population aged 60 years and over has increased continually for each country (except for 1951-1961 in Canada) and has never gone below 6.8\% .

The patterns of the take off of population aging in China (1971-2007) and Canada (1911-1950) are very similar. The only difference is that in China, the take off occurred 60 years later than in Canada (Figure 1). However, the following similarities should be noted. First, both populations experienced an analogous rejuvenation, which preceded the take off of population aging. Second, an almost identical scenario appeared in the two countries: the proportion of persons aged 60 and over was at $7 \%$ when population aging began; and after continuous increase for about 40 years, this proportion reached $11 \%$. And third, during the entire take off period, population aging proceeded 


\section{Figure 1 . Trends of population aging and transition of fertility, China (1950-2050), Canada (1871-2050)}

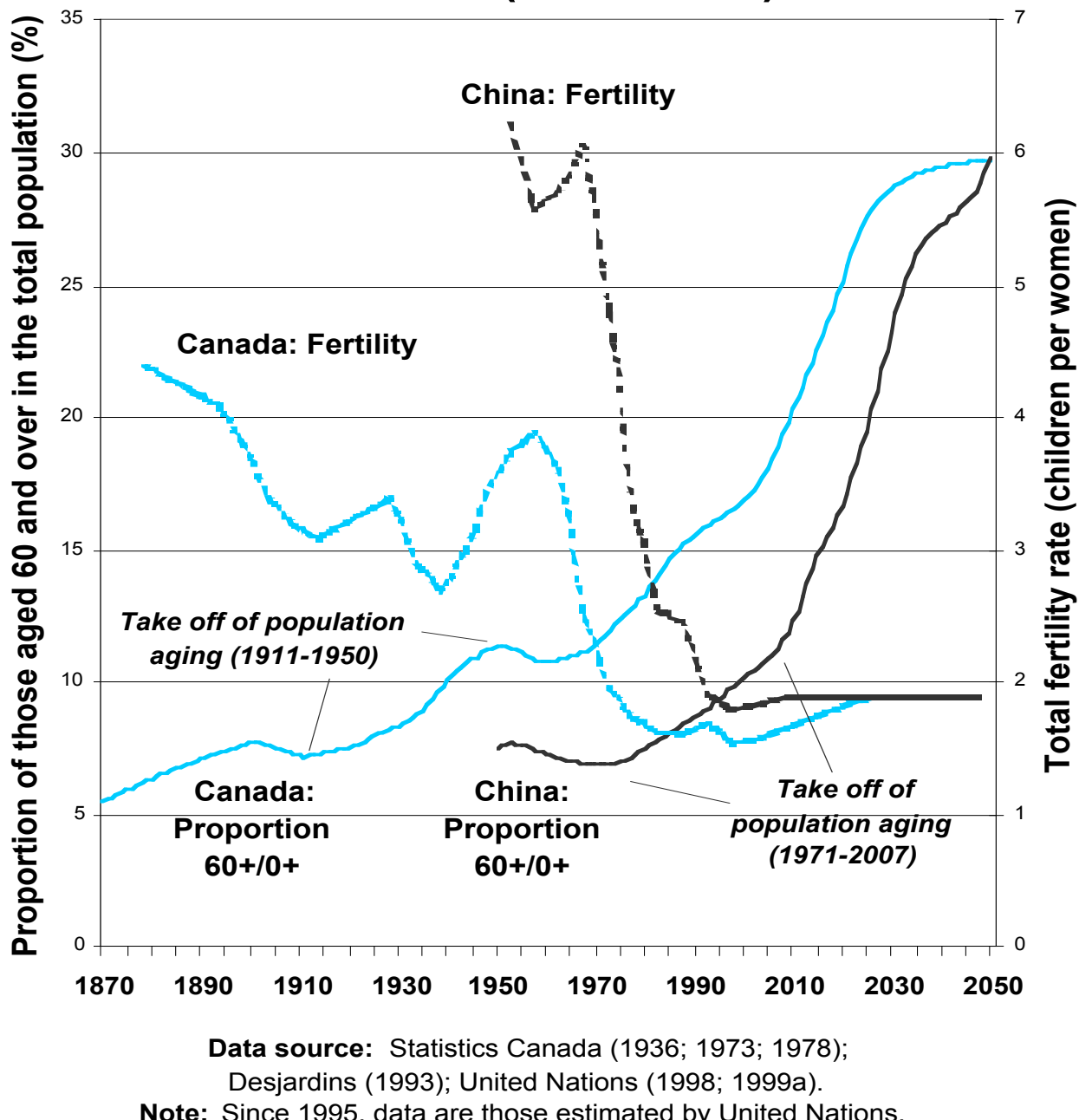

Note: Since 1995 , data are those estimated by United Nations. 
Diversity and Convergence of Population Aging: Evidence from China and Canada

at a regular pace in both countries. The proportion of those aged 60 and over increased, on average, by $1.4 \%$ per year in China and $1.2 \%$ per year in Canada.

The demographic contexts of the take off of population aging in these two countries are also somewhat analogous. The take off of population aging in both Canada (1911-1950) and China (1971-2007) was following an important decline in fertility (Figure 1) and was simultaneous with an increase in the life expectancy (Yan and Chen 1991; Bourbeau et al. 1997).

However, the demographic contexts of the two processes also show some dissimilarities. First, in Canada population aging began in the first half of the 20th century, with significant gains in life expectancy, which are still being made to this day. In China, population aging became apparent only in the 1970s, when the increase in life expectancy had levelled off, after a high rising tide in the 1950s and 1960s (United Nations 1999a). Second, before and during the take off of population aging, there is almost no international migration in China, whereas there is such migration in Canada. For example, from 1911 to 1930, the number of people arriving in Canada ranged from 37,000 to 400,000 annually. In 1913, the immigration rate was 3.9\% (Statistics Canada 1983). Third, since the early 1970 s, China has applied a strict family planning policy, which caused a sharp drop in fertility. At the same time, the population aging process began. This clearly differs from the Canadian situation. And fourth, although the decline in fertility is much more pronounced in China than in Canada, the take off of population aging in the two countries has, for a period of about 40 years, maintained the same trend (Figure 1). This suggests the hypothesis that the total effects of mortality, migration and initial age structure on the take off of population aging were stronger in Canada than in China.

Overall, we observe that the same population aging processes can occur under very different demographic conditions.

\section{Levels of Population Aging}

Before 2050, the level of population aging in China will remain below that of Canada, but the gap will constantly narrow, especially after 2025 (Figure 1). In 1975 , the proportion of those aged 60 and over was $69 \%$ higher in Canada than in China; in 2025, it will be $41 \%$ higher. If this trend continues until 2050, the proportion of those aged 60 and over in China will exceed that in Canada for the first time in history. This proportion reached the $10 \%$ level 59 years later in China than in Canada (2000 in China and 1941 in Canada), whereas the 20\% level will be reached in China within just 16 years of one another (2026 in China and 2010 in Canada). 
Long Mo and Jacques Légaré

Between 1971 and 2050, the level of population aging in China will be higher than in most developing countries, but until 2025 it will be lower than in most developed countries. However, we anticipate that in 2050, it will surpass the levels of at least eight developed countries, including the United States, Canada and Australia (United Nations 1998).

\section{The Speed of Population Aging}

The demographic transition in China has some particular features. The decline in fertility resulting from that country's extraordinary family planning policy and rapid economic growth is striking. It took only twelve years for the Chinese birth rate to drop from $40 \%$ to $20 \%$ (1964-1976) (CPIRC/UNFPA 1995), whereas the same transition took 148 years in France (1761-1909) and 105 years in Germany (1821-1926) (Chesnais 1992).

Generally, a rapid drop in fertility leads to rapid population aging (United Nations 1988).This is the case in China as well. According to UN estimates, for the 60 and over segment of the population to double from $12 \%$ to $24 \%$ will require 141 years in France (1872-2013), 77 years in the United States (19452022) and 37 years in Japan (1977-2014), which is, among the developed countries, the country in which population aging is proceeding most rapidly. However, it will take only 22 years for this same change to occur in China (2010-2032) (United Nations 1956; 1999a). The pace of population aging in China will considerably exceed that of the developed countries for the entire history of their population aging process to the present time.

Between 1975 and 2025, population aging in China will occur more rapidly than in most countries of the world. The speed of population aging, which is defined in this study as the average annual rate of increase of the 60 and over segment of the total population during a given period, was $1.5 \%$ in $1975-2000$ in China, and will be $2.7 \%$ in $2000-2025$. Not only is this rate higher than the average for developed countries ( $0.9 \%$ in $1975-2000$ and $1.4 \%$ in $2000-2025)$, but it is also higher than for many developing countries (an average of $0.9 \%$ and $2.1 \%$ respectively). But it is not the highest in the world. In 2000-2025, the period out of the three 25-year periods between 1975 and 2050 during which population aging in China will be most rapid (2.7\%), there will be 16 countries with a higher speed of population aging, including Singapore (4.1\%), North Korea $(3.2 \%)$, South Korea (3.0\%), Malaysia (3.0\%) and Thailand $(3.0 \%)$. In

Singapore and South Korea, not only the speed, but also the level of population aging will be higher than in China during this period (United Nations 1999b). 
Comparing the speed of population aging in China and Canada, we make the following four observations: First, from the 1990s to 2050, the speed of population aging is clearly higher in China than in Canada, except during 20022008, when the two countries' speeds are similar. Second, two peaks of population aging, with a speed exceeding $3 \%$, will occur in China in 2010-2015 and 2022-2031, respectively. The maximum speed will be $4.1 \%$ in 2013 . This is a phenomenon that has never occurred in Canada. Between 1911 and 2050, the maximum speed of population aging experienced in Canada was $2.6 \%$ in 1940 . Third, in both China and Canada, population aging will increase rapidly during the first half of the $21^{\text {st }}$ century. In Canada, it will reach its peak from 2004 to 2024 , when the speed of population aging will range from $1.5 \%$ to $2.3 \%$ annually. In 2000-2025, among the developed countries Canada will be one of the countries with the most rapid population aging. At this time, in terms of speed of population aging, Canada will be in fourth place among developed counties and in first place among the eight main Western industrial countries (the G-8). In China, the speed of population aging will reach its peak in 20062034 with a rate of $2 \%$ to $4 \%$. Fourth, the speed of population aging fluctuates considerably throughout the population aging process in these two countries.

In both China and Canada, the speed of demographic aging during the first half of the $21^{\text {st }}$ century has close connections with the speed of the fertility decline which occurs during the last half of the 20th century. The total fertility rate decreased from 3.90 children per woman in 1955-1960 to 1.55 in 1995-2000 in Canada whereas from 6.06 in 1965-1970 to 1.80 in 1995-2000 in China. The fertility decline takes a faster pace in China than in Canada. That necessarily led to faster demographic aging in China than in Canada (Figure 1).

Finally, we note that during the 2040s, China will enter into a very particular period of population aging. For one thing, whereas since 1971, Chinese population aging has always been accompanied by an increase in the total population, as of 2039, according to the UN, the Chinese population will start to decrease (United Nations 1998). Thus, the Chinese situation will be similar to that of many developed countries, excluding Canada, but very different from that of other developing countries. For another, as a result of the rebound in the birth rate during the 1980s, in particular in 1985-1990, the rate of increase of the seniors aged 60 and over will again rise significantly in China from 2046 to 2050, after a 13-year decline (2027-2039) and a 6-year stabilization (20402045). This differs from the situation for developed countries overall. So, we again find that Chinese population aging and its context differ not only from those of developed countries, but also from those of developing countries. 
Long Mo and Jacques Légaré

\section{Stages of Population Aging}

During their population aging processes, China (1971-2050) and Canada (19112050) show the same increase in the 60 and over segment of the population, that is, from $7 \%$ to $30 \%$. However, neither the chronology nor the typology of their population aging are similar. Because of these differences, which can be attributed to differences in demographic and socioeconomic conditions, the consequences of population aging differ in the two countries and therefore require different countermeasures.

\section{Two Types of Population Aging Development}

In accordance with population aging intensity, we divide the population aging processes into four stages. If we define $P 60+$ as the proportion of those aged 60 and over within the total population, the four stages of population aging are defined as follows: low population aging $(P 60+<10 \%)$, medium population aging $(10 \% \leq P 60+<20 \%)$, high population aging $(20 \% \leq P 60+<30 \%)$ and extra-high population aging $(P 60+\geq 30 \%)$.

According to this definition, the population aging process considered here is subdivided into three stages: in China, low population aging from 1971 to 1999 , medium population aging from 2000 to 2025 and high population aging from 2026 to 2050; in Canada, low population aging from 1911 to 1940, medium population aging from 1941 to 2009 and high population aging from 2010 to 2050. When we compare the stages of population aging in China and Canada, we make the following four observations (Table 1). First, each of the three stages of population aging appears later and lasts a shorter time in China than in Canada. In particular, Canada spends 68 years in the medium population aging stage, while China spends only 25 years in this stage. Second, during the first half of the $21^{\text {st }}$ century, China will pass through two stages of population aging in succession, that is, medium and high population aging. Canada will take more than a century to go through the same change (1941-2050). Again, this shows the rapidity of population aging in China. Thirdly, at present, population aging in Canada is near the end of the medium stage (1941-2009), while in China, the medium stage has just begun (2000-2025). And fourthly, during the same stage of population aging, China and Canada go through a similar age transition. For example, in the medium population aging stage, the proportion of those aged under 15 declines from $24.8 \%$ to $18.3 \%$ in China and from $27.8 \%$ to $16.7 \%$ in Canada; the population aging index increases from $40.7 \%$ to $106.3 \%$ in China and from $36.7 \%$ to $118.0 \%$ in Canada; the median age rises from 30.0 to 38.9 years in China and from 27.1 to 39.7 years in Canada. Both the direction and the interval of change of these age indicators are similar for the two countries 
Table 1

The Development of Demographic Indicators during Three Stages of Aging for China (1971 - 2050) and for Canada (1911 - 2050)

\begin{tabular}{|c|c|c|c|c|c|c|}
\hline \multirow[t]{2}{*}{ Demographic Indicators } & \multicolumn{2}{|c|}{$\begin{array}{l}\text { Low Ageing } \\
\text { P60 }+<10 \%\end{array}$} & \multicolumn{2}{|c|}{$\begin{array}{c}\text { Medium Ageing } \\
\begin{array}{c}10 \%<=\mathrm{P} 60+<20 \% \\
\text { China }\end{array}\end{array}$} & \multicolumn{2}{|c|}{$\begin{array}{c}\text { High Ageing } \\
\mathbf{2 0 \%}<=\mathbf{P} 60+<\mathbf{3 0} \%\end{array}$} \\
\hline & 1971 & 1999 & 2000 & 2025 & 2026 & 2050 \\
\hline Proportion $60+/ 0+(\%)$ & 6.8 & 10.0 & 10.1 & 19.5 & 20.2 & 29.8 \\
\hline Proportion $0-14 / 0+(\%)$ & 39.8 & 25.3 & 24.8 & 18.3 & 18.1 & 16.3 \\
\hline Ageing Index 60+/0-14 (\%) & 17.2 & 39.4 & 40.7 & 106.3 & 111.4 & 182.5 \\
\hline Median Age (year) & 19.8 & 29.5 & 30.0 & 38.9 & 39.3 & 43.7 \\
\hline Average Speed of Ageing (\%) & \multicolumn{2}{|c|}{1.4} & \multicolumn{2}{|c|}{2.7} & \multicolumn{2}{|c|}{1.6} \\
\hline Proportion $60-69 / 60+(\%)$ & 64.6 & 59.9 & 59.4 & 56.1 & 56.1 & 43.4 \\
\hline Proportion $80+/ 60+(\%)$ & 7.3 & 8.7 & 8.9 & 10.6 & 10.7 & 22.7 \\
\hline Dependency ratio $60+/ 15-59(\%)$ & 12.8 & 15.4 & 15.5 & 31.3 & 32.8 & 55.1 \\
\hline Dependency ratio 0-14/15-59 (\%) & 74.5 & 39.0 & 38.2 & 29.5 & 29.3 & 30.2 \\
\hline \multirow[t]{3}{*}{ Total ratio $0-14$ and $60+/ 15-59(\%)$} & 87.3 & 54.4 & 53.7 & 60.8 & 62.2 & 85.3 \\
\hline & & & \multicolumn{2}{|c|}{ Canada } & & \\
\hline & 1971 & 1999 & 2000 & 2025 & 2026 & 2050 \\
\hline Proportion $60+/ 0+(\%)$ & 7.1 & 10.0 & 10.2 & 19.7 & 20.2 & 29.7 \\
\hline Proportion $0-14 / 0+(\%)$ & 33.1 & 27.9 & 27.8 & 16.7 & 16.6 & 17.0 \\
\hline Ageing Index 60+/0-14 (\%) & 21.6 & 35.8 & 36.7 & 118.0 & 121.7 & 174.7 \\
\hline Median Age (year) & 23.8 & 26.9 & 27.2 & 39.7 & 40.0 & 42.6 \\
\hline Average Speed of Ageing (\%) & \multicolumn{2}{|c|}{1.2} & \multicolumn{2}{|c|}{1.0} & \multicolumn{2}{|c|}{1.0} \\
\hline Proportion $60-69 / 60+(\%)$ & 60.4 & 60.5 & 60.8 & 49.9 & 50.6 & 38.5 \\
\hline Proportion $80+/ 60+(\%)$ & 9.6 & 9.2 & 9.1 & 19.9 & 19.8 & 30.0 \\
\hline Dependency ratio $60+/ 15-59(\%)$ & 11.9 & 16.1 & 16.5 & 31.1 & 32.0 & 55.6 \\
\hline Dependency ratio 0-14/15-59 (\%) & 55.3 & 45.0 & 44.8 & 26.3 & 26.3 & 31.8 \\
\hline Total ratio $0-14$ and $60+/ 15-59(\%)$ & 67.2 & 61.1 & 61.3 & 57.4 & 58.3 & 87.4 \\
\hline
\end{tabular}


Long Mo and Jacques Légaré

during the medium population aging stage.

Furthermore, we find that the demographic implication of population aging in the two countries is not the same, even for an identical population aging stage. First, population aging in China occurs more rapidly than it does in Canada at the same stage. For example, the speed of population aging in China is 1.7 times faster than in Canada during the medium population aging stage. Also, for the same stage of population aging, the elderly Chinese population will always be younger than their Canadian peers. For example, in the medium population aging stage, the proportion of those aged 80 and over within the 60 and over population increases from $8.9 \%$ to $10.6 \%$ in China, but from $9.1 \%$ to $19.9 \%$ in Canada. Moreover, for the same stage of population aging, while the old age dependency ratio is very similar for the two countries, the total dependency ratio is not necessarily similar because of the fluctuation of the youth dependency ratio. For example, in the medium population aging stage, the dependency ratio of those aged 60 and over always remains between $15 \%$ and $31 \%$ in the two countries, while the total dependency ratio ranges from $47 \%$ to $61 \%$ in China and between $55 \%$ and $81 \%$ in Canada ${ }^{2}$.

\section{Population Aging by the Bottom, the Center and the Top}

In studies of the age transition pathways, we frequently see the notions of population aging by the bottom, the center and the top applied (Chesnais 1992). The causes, manifestations and consequences of these three phases of population aging differ and thus it is important to examine their pathways during the population aging process.

Unfortunately, in the literature, the definitions of these notions are often varied or ambiguous (Paillat 1982; Wu 1987; Dittgen and Legoux 1990; Chesnais 1992; Mo 1992; Du 1994; Qiao et al. 1999). This study aims to introduce a new approach to the concepts of population aging by the bottom, the center and the top by setting out precise definitions and original indicators.

We will now give a more specific definition for each one as follows. First, population aging by the bottom is defined as the population aging phase during which the under 15 portion of the total population declines. Second, population aging by the center is defined as the population aging phase during which the 15-59 segment declines. Third, population aging by the top is defined as the population aging phase during which the 60 and over portion increases. 
Consequently, population aging by the bottom, the center and the top is characterized by the relative shrinking of the base and the middle and the relative inflation of the top of the age pyramid, which accounts for the name given to these concepts.

Let us define as follows population aging by the bottom index $\alpha$, population aging by the center index $\beta$ and population aging by the top index $\gamma$ :

$$
\begin{gathered}
\alpha=\left(P c^{\prime}-P c^{\prime \prime}\right) \times 100, \beta=\left(P a^{\prime}-P a^{\prime \prime}\right) \times 100, \gamma=\left(P s^{\prime \prime}\right. \\
\left.-P s^{\prime}\right) \times 100
\end{gathered}
$$

where $P c^{\prime}=$ Proportion of children aged under 15 at the beginning of the year (within the total population, in this case and hereafter), $P c^{\prime \prime}=$ Proportion of children aged under 15 at the end of the year, $P a^{\prime}=$ Proportion of adults aged $15-59$ at the beginning of the year, $P a^{\prime \prime}=$ Proportion of adults aged 15-59 at the end of the year, $P s^{\prime}=$ Proportion of seniors aged 60 and over at the beginning of the year, $P s^{\prime \prime}=$ Proportion of seniors aged 60 and over at the end of the year.

The $\alpha, \beta$ and $\gamma$ indices measure the absolute intensity of population aging by the bottom, the center and the top respectively.

Thus, the $\alpha$ and $\beta$ indices represent the percentage of decline in the proportion of those aged under 15 and those aged 15-59, while the $\gamma$ index represents the percentage of increase in the proportion of those aged 60 and over during the population aging process. Demographically, the $\alpha, \beta$, and $\gamma$ indices clearly convey the intensity of population aging by the bottom, the center and the top. On the one hand, according to our definition, we have the equation $\gamma=\alpha+\beta$, that is, the increase in the proportion of seniors necessarily follows from declines in the proportions of children and adults. In other words, general population aging, which is, population aging by the top, results from the combination of the population aging by the bottom and by the center. On the other hand, the indices accurately reflect the demographic effects of these three types of population aging on the age structure.

The above concepts naturally lead to the following rule: during population aging, if $\alpha>0$, there is necessarily population aging by the bottom, and vice versa; if $\beta>0$, there is necessarily population aging by the center, and vice versa; if $\gamma>0$, there is necessarily population aging by the top, and vice versa.

Another benefit of establishing the $\alpha, \beta$, and $\gamma$ indices is that they enable us to measure in a comparable way the intensity of these three types of population 
aging. For example, if $\alpha>\gamma(\alpha>0$ and $\gamma>0)$, this means that the intensity of population aging by the bottom is stronger than that of population aging by the top. Thus we can compare population aging by the bottom, the centre and the top quantitatively, not only among each other for the same population, but also between different populations. Therefore, we can say that there is population aging primarily by the bottom when $\operatorname{Max}(\alpha, \beta, \gamma)=\alpha$, population aging primarily by the center when $\operatorname{Max}(\alpha, \beta, \gamma)=\beta$, and population aging primarily by the top when $\operatorname{Max}(\alpha, \beta, \gamma)=\gamma$.

With the help of the above definitions and indices, which give us a new approach to population aging by the bottom, the center and the top, we will now compare these three types of population aging in China and Canada.

First, using the $\alpha, \beta$ and $\gamma$ indices, for the first time we are able to evaluate quantitatively and graphically the timetable and the intensity of population aging by the bottom, the center and the top during the population aging processes in China and in Canada (Figure 2).

Second, in terms of the three types of population aging concerned, the chronology of population aging in China is clear. The first stage takes place between 1974 and 2008, during which time the population aging is evidently primarily by the bottom. During this period, population aging by the bottom is very strong, especially in 1976-1988 and 2000-2005 $(0.5<\alpha<1.1)$, while population aging by the top is weak $(0<\gamma<0.3)$ and population aging by the center is negative. This shows that in this stage, population aging is due mainly to the decline in the birth rate. From 2009 to 2042, the type of population aging changes and it is primarily by the top. In other words, population aging is due chiefly to the rapid increase in the number of older people. In this second stage, which is clearly differentiated from the first, the three types of population aging coincide, but population aging by the top dominates, especially in 2011-2015 and 2021-2034 $(0.5<\gamma<0.8)$. This is in contrast to the Canadian situation. In Canada, the chronology of population aging primarily by the bottom, the centre and the top is distinct before 1982, but murky from then until 2050 .

Third, both China and Canada will soon be facing population aging simultaneously by the top and by the center, accompanied by a considerable increase in the number of seniors and a downward trend in the rate of increase of adults. For example, the population aging by the top and population aging by the center indices will reach their peak simultaneously in China in 2011-2036 $(0.4<$ $\gamma<0.8$ and $0.3<\beta<0.6$ ). 
Figure 2.

Aging by the bottom, the centre and the top, China (1971-2050), Canada (1911-2050)

Source: Calculated from Statistics Canada $(1936 ; 1973)$ and United Nations (1998) data.
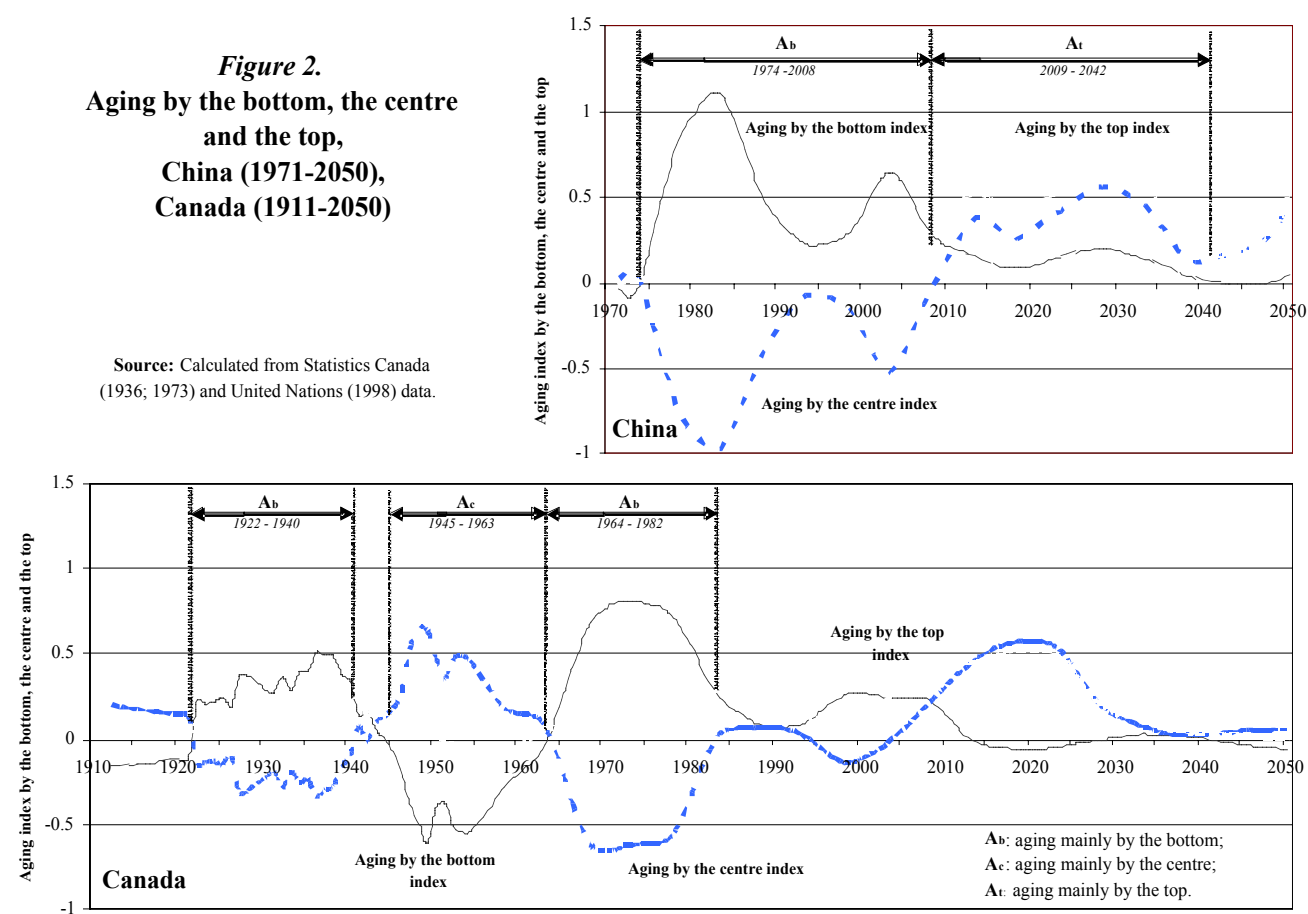
Long Mo and Jacques Légaré

Fourth, it was thought that population aging by the bottom would be much stronger in China, owing to its strict family planning, than in developed countries. We find that this type of population aging was very strong in China in 1976-1988 $(0.5<\alpha<1.1)$, but Canada also had very strong population aging by the bottom in $1967-1980(0.5<\alpha<0.8)$. In fact, a similar decline in the number of births occurred during the above-mentioned periods respectively in the two countries (United Nations 1999a), although the socioeconomic causes were different.

Fifth, our results go against the widely held belief that population aging by the top is more pronounced in developed countries, where life expectancy is higher, than in developing countries. According to our calculations, for the 25-year period in which population aging by the top will reach its maximum, that is, 2011-2035 in China and 2003-2027 in Canada, the sum of the annual population aging by the top index for the period studied is higher in China $(\Sigma \gamma=13.9)$ than in Canada $(\Sigma \gamma=10.9)$. We can attribute this, in part, to the fact that the wave of births which hit China in 1950-1958 and 1962-1973, and Canada in 1946-1965, and which will soon give rise to a influx of seniors, was stronger in China than in Canada (Statistics Canada 1983; Yuan et al. 1991).

Sixth, population aging by the bottom, the center and the top will occur simultaneously in China in 2009-2050, reaching the maximum in the same period 2020-2035. However, this never happens in Canada. And it is precisely this coincidence of the three types of population aging that largely explains the extreme rapidity of population aging in China. As our study shows, the combined effect of population aging by the bottom, the center and the top has a great influence on the speed of population aging.

Finally, in contrast to the theoretical expectation that population aging generally goes from population aging primarily by the bottom, to population aging primarily by the center and then by the top (Chesnais 1992), population aging primarily by the center never occurs in China, and in Canada the three types of population aging appear alternatively and recurrently. Moreover, population aging primarily by the top is not evident in Canada. This, again, shows the diversity of the population aging process.

\section{Population Aging and Development}

Population aging is not an isolated process. On the contrary, it is believed to be closely tied to changes in socioeconomic conditions. In this section, we will look at the relationship between demographic aging stages and socioeconomic development stages. For this purpose, we analyze the scatterplot of the United 
Nations Human Development Index and the proportion of those 65 and over in 1998 in 144 countries whose population exceeds one million inhabitants. Observing the layout, we see that these two variables appear to be correlated and their combined distribution takes the form a curve (Figure 3). An estimate of the curve yields the following regression model:

$$
P A=0.0238+0.1312 * H D I-0.5265^{*} H D I^{2}+0.5671^{*} H D I^{3}
$$

where,

$$
\begin{aligned}
& P A=\text { Proportion of those aged } 65 \text { and over in the total population } \\
& H D I=\mathrm{UN} \text { Human Development Index }
\end{aligned}
$$

Figure 3. Aging and development: a regression analysis among 144 countries $^{*}$ and the situation of China and Canada, 1998 ( ${ }^{*}$ All the countries of the world whose population exceeds one million inhabitants )

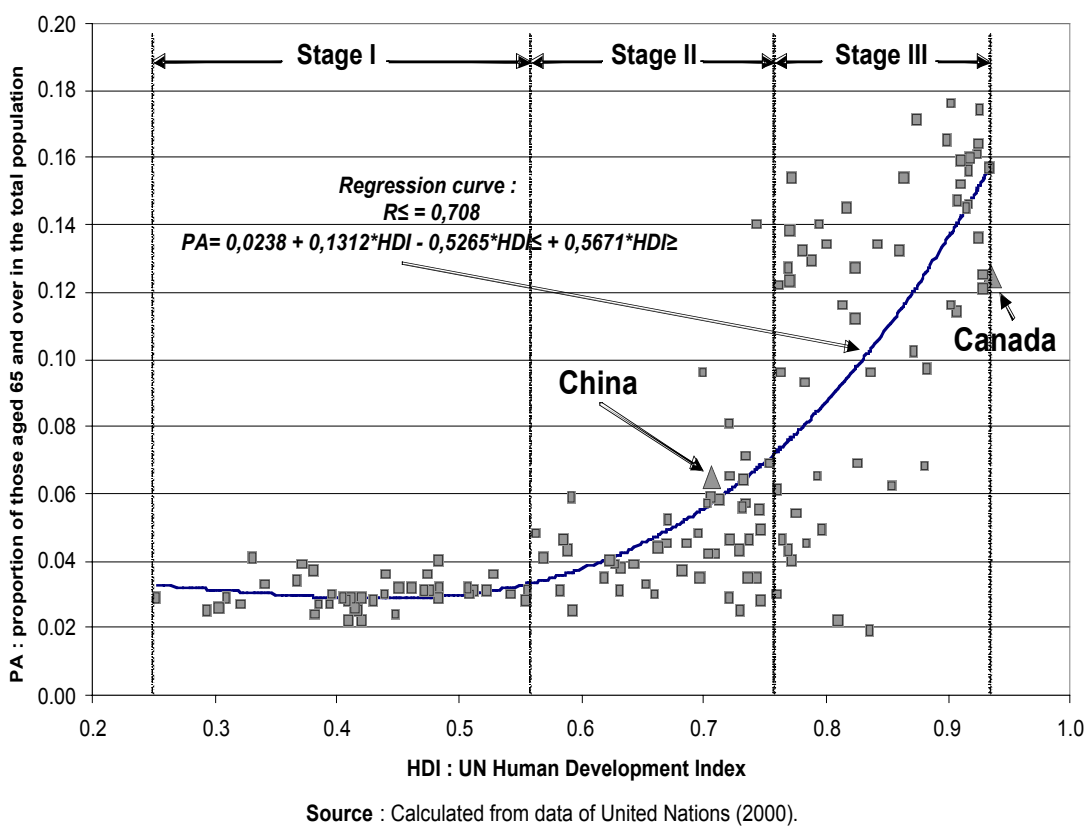


Long Mo and Jacques Légaré

From this regression analysis, we are led to make the following comments:

First, the regression curve obtained here has a high goodness of fit $\left(R^{2}=0,708\right)$. This shows that, in 1998 and internationally, there was a close relation between the level of population aging and the level of development. On the one hand, population aging is the natural product of the demographic transition, and fertility change is the major factor (United Nations 1988; George et al. 1991; Du 1994; Mo 2002). On the other hand, there is extensive literature linking socioeconomic development and fertility change. Even in China where the effect of the policy of family planning was significant during the three last decades of the $20^{\text {th }}$ century, the rapid economic growth also played a significant role in the fast fall of fertility during this period (Poston and Gu 1987; Lavely and Freedman 1990; Yuan et al. 1991; Zhang 1998). A study subsidized by China Social Science National Funds shows that, in 1971-1998, 54\% of Chinese fertility fall are explained by the factor of family planning and $46 \%$ by the factor of socioeconomic development (Zha et al. 2000). Indeed, according to the theory of demographic transition, the degree of advancement of the demographic transition and the stage of socioeconomic development are closely associated (Chesnais 1992). Thus, we believe that generally speaking, population aging stage is dependent upon development stage. Our regression analysis confirms this relationship quantitatively.

Secondly, according to our calculations, there is a high coefficient of correlation between the two variables $P A$ and $H D I(R=+0,747)$. Here is the T-test:

$$
\mathrm{T}^{*}=\left(\mathrm{R} \_\sqrt{n-2}\right) / \sqrt{1-R^{2}}=(0.747 \sqrt{144-2}) / \sqrt{1-0.747^{2}}=13.39
$$

Where, $\mathrm{n}=$ Number of countries in the regression analysis.

When $\alpha=0.05, \mathrm{~T}_{\alpha \mathrm{R}}=1.98$. Because $\mathrm{T}^{*}>\mathrm{T}_{\alpha \mathrm{R}}$, so, it is judged that there is an obvious positive correlation between $P A$ and $H D I$. In other words, as a general tendency, the more the level of development is high, the more the level of population aging is high. However, as we will see below, the strength of this correlation varies from one stage of development to another, and it is difficult to pinpoint for low levels of development.

Third, for each development stage, population aging presents different characteristics. China and Canada are in different development stages. It is this difference that leads to the differences in population aging between the two countries.

After a general analysis of the distribution of the $P A$ and $H D I$ variables in Figure 3 , we divide the modern development process into three stages, in relation to the 
$H D I$. The first stage is defined as $0.25 \leq H D I<0.56$. We can summarize the population aging characteristics of the 42 countries found in this stage in 1998 as follows: (1) The proportion of older people is very low -- the average $P A$ in these countries is only $3.0 \%(V \sigma=0.113)$. (2) Population aging is either not present or its pace is very slow -- the average annual rate of increase in the proportion of those aged 65 and over in 1998-2015 ( Vpa $)$ is only $0.15 \%(V \sigma=$ 4.626), and there are 17 countries in which the population will become even younger. (3) There is very little correlation between population aging and development -- the coefficient of correlation between $P A$ and $H D I$ is very low $(R=0.157)$. The second stage: $0.56 \leq H D I<0.76$. There are 47 countries in this stage, which have the following population aging characteristics: (1) The proportion of seniors in most of these countries is somewhat higher than in the previous stage -- the average $P A$ is $4.9 \%(V \sigma=0.281)$. (2) Population aging has begun in almost all these countries and in some, has even accelerated -- the average $V p a$ is $1.12 \%(V \sigma=0.639)$; (3) In comparison with the previous stage, the dependency between $P A$ and $H D I$ is stronger -- $R=0.337$. The third stage: $0.76 \leq H D I<0.94$. Population aging in the 55 countries in this stage is characterized as follows: (1) The proportion of seniors is generally higher than in the countries in the two earlier stages -- the average $P A$ is $11.3 \%(V \sigma=$ 0.332 ). (2) The speed of population aging is generally high -- the average $V p a$ is $1.75 \%(V \sigma=0.470)$. (3) The correlation between population aging and development is the strongest of all the three stages -- $R=0.560$.

According to this analysis, in 1998 China and Canada were in the second and third stage respectively, and, as we mentioned earlier, these stages have different population aging characteristics. On the HDI, Canada is in first place in the world, while China is in ninety-ninth place. This large socioeconomic difference leads to differences between the two countries in the causes, processes and consequences of population aging. An important fact to note is that China will soon enter the third development stage. According to the general rule illustrated above, the influence of the development factor on population aging will then be stronger.

Fourth, in comparison with other countries, the population aging process in China precedes the development process in 1998, while in Canada, the situation is reversed. In Figure 3, China's indicator is just above the regression curve, while Canada's is clearly below. This shows the precocity in China and lateness in Canada of population aging. Indeed, compared to other countries with a similar development level $(H D I)$, the level of population aging $(P A)$ is higher in China and lower in Canada. To explain the causes of this situation, we make the following hypothesis: the observed phenomena are largely the result of strict family planning in China and significant immigration in Canada. However, in contrast to the widely held perception, the precocity of population aging in 
Long Mo and Jacques Légaré

China is not very great. This can be explained by rapid economic growth in the last two decades and the relatively high life expectancy of the population compared to other developing countries. These two factors contribute to the rise of China's Human Development Index.

\section{The Convergence of Population Aging}

While the preceding comparison of the patterns and the stages of population aging process in China and Canada confirms the diversity of population aging process, the next section bears witness to the convergence of the population aging process.

\section{The Similarity of Age Structure in the Middle of the $21^{\text {st }}$ Century}

Up to now, the age structures of the Chinese and Canadian populations have been notably different. However, we note that, according to the projections of the United Nations, the populations of the two countries will move towards a similar age structure in 2050. At this time, not only the proportion of persons aged 60 years and over, but also other age indicators such as the median age, the proportions of children and adults as well as the accumulated proportion of the population by age of the two countries are similar (Figure 1 and Mo 2002). This similarity is illustrated by the population pyramids of the two countries for 2050 (Figure 4).

\section{The Similarity of the Age Transition during Population Aging}

At the beginning of the process of population aging, i.e. in 1971 in China and in 1911 in Canada, the age structures of the two countries are similar. Since then and up to 2050, during the process of population aging, the population pyramids of the two countries show the same historical transformation, passing from a "typical pyramid" whose base is particularly broad to a "rhombus" whose centre inflates and finally to a "rectangle" in which the widths of surfaces of all the quinquennial age groups before the age of 70 years approach each other (Figure 4). This shows that, during the process of population aging, a similar age transition is carried out in the two populations. 
Figure 4. Transformation of the population pyramids during the process of aging, China (1971-2050), Canada (1911-2050)

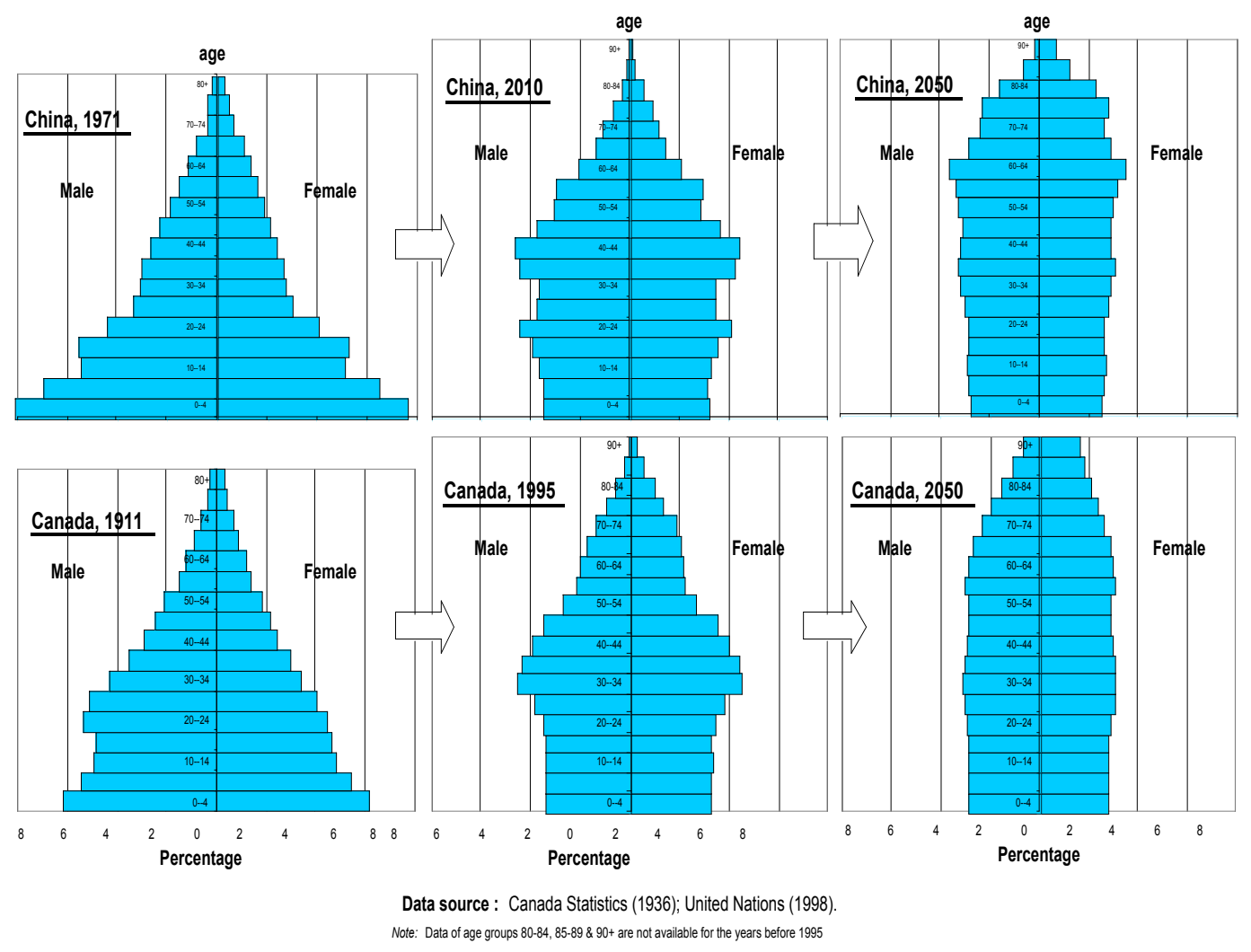


Long Mo and Jacques Légaré

We also note that, as the level of population aging converges, its demographic consequences, for example, dependency ratio will get closer. Currently, the dependency ratio of those aged 60 and over to those aged 15-59, the dependency ratio of those aged 0-14 to those aged 15-59 and the total dependency ratio are different in China and in Canada, while in the middle of the $21^{\text {st }}$ century, the three ratios will reach, respectively, about same level in the two countries studied (United Nations 1998).

\section{Conclusion}

Demographic aging is a relentless and universal phenomenon. According to demographic projections of the United Nations, after the widespread appearance of population aging in the developed countries during the last half of the $20^{\text {th }}$ century, this phenomenon will occur, for the first time in history, in almost all the developing countries in the five decades to come. Moreover, the rhythm of population aging will be notably faster in the developing countries than in the developed countries (United Nations 1998). Is this a sign of population aging convergence between developing countries and developed countries? Taking into account the convergence of the demographic transition and close relationship between demographic transition and population aging (Chesnais 1992), this is not impossible. By comparing the situation in China and in Canada, the present study bears witness to the convergence of population aging between these two countries. In spite of a time lag of sixty years, the demographic aging of China and Canada converge in the middle of the $21^{\text {st }}$ century: as well both age indicators and the population pyramids show that the age structures of the two countries will be similar at that time.

However, the two countries achieve the same age transition through the two different paths. The present study also testifies to the diversity of population aging. We find in this paper that the similarities and dissimilarities of the population aging process between China and Canada are linked, and that the disparity of the population aging process between the two countries studied is considerably more marked than that observed among the developed countries. In substance, the demographic aging processes of China and Canada belong to two types.

Through a comparison with the Canadian situation, we show the singularity of the population aging process in China as follows: (1) Late take off. (2) Extreme rapidity. (3) A relatively low level of population aging, but with the gap tending to narrow. (4) Different demographic implication of population aging. For example, the older Chinese population is still younger than the Canadian one for 
the same level of population aging. (5) Very strong population aging primarily by the bottom and subsequently by the top. (6) Precocity of population aging in relation to development. Furthermore, it should be borne in mind that in China rapid population aging takes place in the socioeconomic conditions of a developing country.

In fact, the diversity and the convergence of population aging result from the diversity and the convergenge of the demographic transition. The principal determinant consists in the transition of fertility. As illustrated by Figure 1, the fertility decline came later, but with greater speed in China than in Canada. Consequently, Chinese population aging appeared later but with a faster speed. China and Canada will face an extremely rapid demographic aging during the first half of the $21^{\text {st }}$ century. This coincidence occurs in the context that a remarkable fertility decline came successively in these two countries during the last half of the 20th century. As for the dynamics of the divergence of population aging, we propose that it results primarily in the convergence of the demographic transition, especially that of the fertility transition. Although past fertility levels and trends are completely different between China and Canada, the two countries total fertility rate already got closer at the threshold of the $21^{\text {st }}$ century and they will converge towards 1.9 child per woman during the first half of the $21^{\text {st }}$ century (United Nations, 1999a). Briefly, China and Canada will achieve the same fertility transition through two distinct pathways. This is the key reason for which these two countries will achieve the same age transition through two different paths.

\section{Acknowledgements:}

An earlier version of this paper was presented at the World Congress of Gerontology, Vancouver, 1-6 July 2001. The authors gratefully acknowledge financial support from the Universite de Montréal and from the Social Sciences and Humanities Research Council of Canada. They also thank the reviewers for their comments and suggestions. 
Long Mo and Jacques Légaré

\section{End Notes:}

1. The definition of elderly varies according to the time and the place. Nowadays, it is the norm in Canada that those aged 65 and over are considered elderly whereas in China it is those aged 60 and over. In order to ensure international comparability, as in the recent United Nations report on world population aging (2002), the present study uses primarily age 60 as the age threshold for elderly. However, we use age 65 as the age threshold for elderly when we conduct the analysis of the relations between demographic aging and socioeconomic development, because this analysis rests on the Human Development Report of United Nations (2000) in which the data with an age threshold of 60 are not available.

2. To note that the total dependence ratio does not necessarily reach its maximum and its minimum at the beginning or the end of the medium population aging stage. Consequently, the intervals of variation of this indicator shown here are not presented in Table 1.

\section{References}

Bourbeau, R., J. Légaré and V. Emond. 1997. New Birth Cohort Life Tables for Canada and Quebec, 1801-1991. Ottawa: Statistics Canada.

Chenais, J.-C. 1992. The Demographic Transition, Stages, Patterns, and Economic Implication. Oxford: Clarendon Press.

CPIRC (China Population Information and Research Centre)/UNFPA (United Nations Population Fund). 1995. Data User Service Series No. 2: Fertility Data of China. Beijing: China Population Publishing House.

Desjardins, B. 1993. Population Ageing and the Elderly. Ottawa: Statistics Canada.

Dittgen, A. and Legoux, Luc. 1990. "Vieillissement par le haut et par le bas: l'exemple de la France," in: Loriaux, Michel et al. (eds.). Populations âgées et révolution grise. Louvain-la-Neuve, Éditions CIACO.

Du, P. 1994. Study on the Process of Population Aging in China. Beijing: China People's University Press. 
George, M. V. et al. 1991. "Effects of fertility and international migration on changing age composition in Canada," Statistical Journal of the United Nations ECE 8 (1): 13-24.

Lavely, W. and R. Freedman. 1990. "The Origins of the Chinese Fertility Decline," Demography 27 (3): 357-367.

Loriaux, M. 2002. "Vieillir au Nord et au Sud : convergences ou divergences?" in: F. Gendreau, D. Tabutin and M. Poupard (eds.). Jeunesses, vieillesse, démographies et sociétés. Louvain-la-Neuve/Paris: Académia-Bruylant/ L'Harmattan.

Mo, Long. 1992. Aged Population Statistics. Nanning: Guangxi People’s Press.

Mo, Long. 2002. Le vieillissement de la population en Chine et au Canada: deux voies pour une même destination? Ph.D. dissertation, Department of Demography, University of Montreal.

Mo, L. and J. Légaré. 2002. "La diversité des processus du vieillissement démographique : les exemples de la Chine et du Canada," in: F. Gendreau, D. Tabutin and M. Poupard (eds.). Jeunesses, vieillesse, démographies et sociétés Louvain-la-Neuve/Paris: AcadémiaBruylant/L'Harmattan.

Mo, L. and J. Légaré. 2003. "Les politiques de fécondité en Chine et d'immigration au Canada : une étude comparée de leurs impacts sur le vieillissement," Cahiers québécois de démographie, forthcoming.

Paillat, P. 1982. Vieillissement et vieillesse. Paris: Presses Universitaires de France.

Poston, D. L. and B.-Ch. Gu 1987. "Socio-economic Development, Family Planning, and Fertility in China," Demography 24 (4): 531-551.

Qiao, X. et al. 1999. "Population aging in China: A century-end perspective," Population Research 23 (6): 28-37.

Statistics Canada. 1936. Seventh Census of Canada, 1931, Volume I, Summary. Ottawa: Dominion Bureau of Statistics.

Statistics Canada. 1973. Population 1921-1971: Revised Annual Estimates of Population, by Sex and Age group, Canada ad the Provinces. Ottawa: Statistics Canada. 
Long Mo and Jacques Légaré

Statistics Canada. 1978. La Statistique de l'état civil, Volume I - Naissances (1975-1976). Ottawa: Statistics Canada.

Statistics Canada. 1983. Historical Statistics of Canada (second edition). Ottawa: Statistics Canada.

United Nations. 1956. The Aging of Populations and its Economic and Social Implications. New York: United Nations.

United Nations. 1988. Economic and Social Implications of Population Aging. New York: United Nations.

United Nations. 1998. World population 1950-2050 (The 1998 revision, magnetic tape data sets, annually). New York: United Nations.

United Nations. 1999a. World Population Prospects - The 1998 Revision, Volume I: Comprehensive Tables. New York: United Nations.

United Nations. 1999b. World Population Prospects - The 1998 Revision, Volume II: The Sex and Age Distribution of the World Population. New York: United Nations.

United Nations. 2000. Human Development Report 2000. United Nations. www. undp.org.

United Nations. 2002. World Population Ageing 1950-2050. United Nations. www.un.org.

Wu, C.-P. 1987. Introduction to Population Aging. Shengyang: Liaoning People's Press.

Yan, R. and Sh.-L. Chen. 1991. "Studies on life expectancy and mortality rates by age in China for the last 40 years," Population Science of China 1991 (2): 1-10.

Yuan, R.-X. et al. 1991. The Population of China Series: National Volume. Beijing: China Finance and Economics Press.

Zha, R.-Zh. et al. 2000. "Prospects for China's Population Problems in the $21^{\text {st }}$ Century," Population Research 24 (1): 28-37. 


\section{Diversity and Convergence of Population Aging: Evidence from China and Canada}

Zhang, F.-Y. 1998. "A Study of the Birth Rate and Natural Population Growth in China in the 1990s - A comprehensive Analysis of the Impact on the Population of Country Level Social, Economic, Ethnic and Child Bearing Policies," Social Sciences in China 1998 (4): 97-112. 